\title{
Could be Used an Immune Phenotypes of Circulating Endothelial Progenitor Cells as a Biomarker of Cardiovascular Disease?
}

\author{
Alexander E. Berezin, Professor, MD, PhD \\ Consultant of Therapeutic Unit, Private Hospital "Vita-Center", Zaporozhye, Ukraine \\ Consultant of Therapeutic Unit, Department of Internal Medicine, Medical University, \\ Zaporozhye, Ukraine
}

\begin{abstract}
Endothelial progenitor cells (EPCs) are recognized a circulating pool of primitive endothelial precursors of different origin from bone marrow or peripheral tissues, which express on their surface endothelial markers, i.e. CD45-, CD34+, CD144+, CD309 etc. There are at least two types of EPCs: early outgrowth EPCs and late outgrowth EPCs, which distinguish each other their structure, function, expression of CD markers and gene expression. Whether various immune phenotypes of circulating EPCs may play similar role in endothelial homeostasis acting as endogenous repair system is not fully understand. However, both immune phenotype EPCs are involved in the pathogenesis of CV diseases across all stages of CV continuum. The short commentary is depicted the possibility to use a dysfunction of different EPCs as a predictive biomarker of $C V$ disease development with promising discriminative value to relate to $C V$ clinical outcomes.
\end{abstract}

Keywords: cardiovascular disease; endothelial progenitor cells, prognosis; outcomes.

Cardiovascular (CV) diseases including atherosclerosis, arrhythmia, myocardial infarction, heart failure remains to be a main cause of death worldwide. Recent animal and clinical studies have shown that an endothelium, which is involved in the pathogenesis of CV diseases across all stages of CV continuum, could coordinate both mutual counteracting processes, i.e. vascular injury and reparation $[2,3]$. The endothelial progenitor cells (EPCs), which enhance angiogenesis, neovascularization and vascular function, act, as endogenous repair system and play a pivotal role in the endothelium homeostasis $[4,5]$.

Pioneer investigations of Asahara et al $[6,7]$ have been shown that EPCs located in a bone marrow and probably in peripheral tissues are able to migrate into injury sites and directly turning into mature endothelial cells and even into smooth muscle cells shaping new vessels. Authors reported that EPCs dramatically enhance the angiopoetic capacity including cell proliferation, migration, and tubule-like formation as well as promote tissue reparation with a reduction in fibrotic tissue and improved neovascularization via significant suppression of inflammatory cell recruitment. Although mechanism of EPC-mediated reparation is not fully understood, it has been found that the EPCs may synthase and secrete a broad spectrum of angiopoetic factors, i.e. vascular endothelial growth factor (VEGF), stromal-cell-derived factor-1 (SDF-1), and fibroblast growth factor (FGF). These factors involve into an attenuation of angiogenesis and vascularization through epigenetic mechanisms affecting as endothelial precursors, as more mature endothelial cells [8]. Acting through several intracellular prosurvival regulators (hypoxia induced factor- $1 \alpha$, Akt / eNOS signaling) EPC-depending cytokines mediate cytoprotective effects on the target cells in injured tissues [9].

Recent studies have revealed the CV risk factors, i.e. diabetes, obesity, impaired fasting glucose, hypothyroidism, hyperuricemia, may directly induce lowering EPC number and weak their functionality that has determined as EPC dysfunction [10-13]. In a result, EPC dysfunction precedes manifestation of endothelial dysfunction and $\mathrm{CV}$ diseases through worsening vascular repair capability $[9,14]$. On the other hand, EPC dysfunction could appear prior CV diseases and even CV risk factors, although there is no a clear explanation of the phenomenon [14-16]. Probably epigenetic dysregulation is a main cause leading to both lowered number and weak function of EPCs beyond influencing conventional CV risk factors [17]. Taken together all these facts suggest that various CV 
risk factor may impact on structure, function, an ability to colony forming and differentiation of different immune phenotypes of EPCs in a distinguished manner.

Recent studies performed ex-vivo with cultured EPCs allow recognizing two different phenotypes of EPCs labelled as early outgrowth EPCs and late outgrowth EPCs and isolated from similar source having similar markers expressing on their surfaces, i.e. CD144, CD309 (VEGF receptor - VEGFR), and CD45 [18, 19]. Late outgrowths EPCs may easily shape colony of endothelial cells than early outgrow EPC. Moreover, late outgrowths EPCs were able to produce more nitric oxide and got better attenuation of capillary structure when compared to early EPC [18]. Consequently, early outgrow EPCs may produce slightly broad spectrum of pro-angiogenic and angiopoetic cytokines including VEGF and interleukin (IL)-6 [19, 20]. Additionally, there was a considerable difference between early outgrow EPCs and late outgrow EPCs in gene expression profiles that affect their ability to express some intracellular signal systems, i.e. stem cell factor (SCF)/c-Kit, angiopoietin-1/Tie2, and SDF1/CXCR4 [21]. It has been postulated that the difference in gene expression in EPC populations could enhance their ability to realize variable effects toward secretion and differentiation. However, nowadays we do not exactly know whether both immune phenotypes of EPCs distinguishing their ability to shaping endothelial cell colony in culture. In this context, cytokine-triggered low intense inflammation as a common pathogenetic factor of $\mathrm{CV}$ diseases may up-regulate an expression of various genes leading to worsening survival ability of EPCs and number depletion of ones [22].

Indeed, an exhaustion of circulating number of EPCs labeled as CD34+ and / or CD133+, AC133+, endothelial cell markers (CD309, CD31, CD 144) was found in patient with atherosclerosis, cardiac dysfunction, myocardial infarction / acute coronary syndrome, hypertension, diabetes mellitus, thyroid dysfunction [14, 15, 23-25].

However, there are several controversies regarding the role of different immune phenotypes of EPCs in tissue reparation. Koller et al (2016) [23] reported that EPCs, defined as triple-positive cells with phenotype CD34+CD45-CD309+, was a significant and independent inverse predictor of mortality of heart failure as ischemic as well as non-ischemic etiology. There is evidence that the number of circulating non-hemopoetic EPCs with immune phenotypes CD14+CD309+ and CD14+CD309+Tie2+ could demonstrate more pretty discriminative value in ischemia-induced cardiac failure than CD45+ CD309+ EPCs [24]. Therefore, lowered number of CD14+CD309+Tie2+ EPCs may suggest shaping systolic cardiac dysfunction, whereas hemopoetically originated EPCs did not exhibit predictive value in left ventricular hypertrophy and isolated diastolic function $[14,25]$.

In population of the asymptomatic coronary artery disease patients increased circulating number of CD45-CD34+ EPCs and a reduced non-classical EPC' population defined as CD14+CD309+ and CD14+CD309+Tie2+ were found [26]. Interestingly, there was an association between number of non-classical phenotype EPCs and diabetes mellitus, highly sensitive C-reactive protein, and Agatston score index , whereas CD45-CD34+ EPCs' number did not exhibit similar relations [26]. Moreover, incorporation of number of CD14+CD309+Tie2+ EPCs as a predictive biomarker into biomarker risk score for cumulative CV events in heart failure patients may sufficiently improve sensitivity, specificity, reliability and final discriminative value of the scale [27]. Yang et al [28] reported that count of circulating EPCs with immune phenotype CD34-/CD133+/KDR+ (VEGFR+) may exhibit osteogenic potential and strong correlates with coronary atherosclerosis at the early stage. Similar results were received by Berezin A et al (2016) [29] in diabetic cohort. Authors reported that lowered number of non-classical EPCs with immune phenotypes CD14+CD309+ and CD14+CD309+Tie2+, but CD34+ subsets of EPCs did not associated well with biomarker of atherosclerosis in individuals with type 2 diabetes mellitus. Finally, EPCs with classical CD34+CD133+CD309+ phenotype and non-classical CD34+Tie2+ phenotypes may sufficiently distinguish each other in their ability to predict $\mathrm{CV}$ diseases and exhibit discriminative value for clinical CV outcomes [30, 31].

Whether increased number of EPCs with various non-classical phenotypes in individuals suspected to atherosclerosis could reflect the risk of all-cause mortality is not fully understood. Further large clinical trials are required to explain the role of various immune phenotypes of circulating EPCs as a biomarker of $\mathrm{CV}$ disease with promising predictive value.

\section{CONCLUSION}

CV disease development associates with exhausting number of circulating EPCs and / or lowered functional ability of ones. CV risk factors are able to modulate innate features of circulating EPCs 
with different immune phenotypes, which correspond to a risk of CV events and CV mortality. However, there is no clear evidence regarding pretty predominance in useful of measurement of immune phenotypes of EPCs to predict CV disease that requires more clinical investigations.

\section{REFERENCES}

[1] Roger VL. Cardiovascular diseases in populations: secular trends and contemporary challengesGeoffrey Rose lecture, European Society of Cardiology meeting 2014. Eur Heart J. 2015; 36(32): 2142-6.

[2] Wong VW, Crawford JD. Vasculogenic cytokines in wound healing. BioMed Research International. 2013; 2013:11

[3] Giacobbe DT, Murray MJ. Vascular disease and inflammation. Anesthesiology Clinics of North America. 2004; 22(2):183-197.

[4] Abe Y, Ozaki Y, Kasuya J, Yamamoto K, Ando J, Sudo R, Ikeda M, Tanishita K. Endothelial progenitor cells promote directional three-dimensional endothelial network formation by secreting vascular endothelial growth factor. PLoS One. 2013; 8(12):e82085.

[5] Berezin A. "Impaired immune phenotype" of endothelial cell-derived microparticles: the missed link between diabetes-related states and cardiovascular complications? Journal of Data Mining in Genomics \& Proteomics. 2016; 7(2): 195-197

[6] Asahara T, Murohara T, Sullivan A, Silver M, van der Zee R, Li T. et al. Isolation of putative progenitor endothelial cells for angiogenesis. Science. 1997; 275:964-967

[7] Asahara T. Endothelial progenitor cells for vascular medicine. Yakugaku Zasshi. 2007; 127(5):841-5.

[8] Kim H, Kim S, Baek SH, Kwon SM. Pivotal Cytoprotective Mediators and Promising Therapeutic Strategies for Endothelial Progenitor Cell-Based Cardiovascular Regeneration. Stem Cells Int. 2016; 2016: 8340257.

[9] Berezin AE. Endothelial progenitor cells dysfunction and impaired tissue reparation: The missed link in diabetes mellitus development. Diabetes Metab Syndr. 2016. doi: 10.1016/j.dsx.2016.08.007.

[10] Berezin AE, Kremzer AA, Martovitskaya YV, Samura TA, Berezina TA. Serum Uric Acid Predicts Declining of Circulating Proangiogenic Mononuclear Progenitor Cells in Chronic Heart Failure Patients. J Cardiovasc Thorac Res, 2014;6(3):153-62

[11] Berezin AE, Kremzer AA. Circulating endothelial progenitor cells as markers for severity of ischemic chronic heart failure. J Card Fail. 2014; 20 (6): 438-447.

[12] Berezin A. Metabolic memory phenomenon in diabetes mellitus: achieving and perspectives. Diabetes \& Metabolic Syndrome: Clinical Research \& Reviews. 2016; [Epub ahead of print] DOI: 10.1016/j.dsx.2016.03.016.

[13] Berezin AE, Kremzer AA, Berezina TA, Martovitskaya YV, Gronenko EA. Data regarding association between serum osteoprotegerin level, numerous of circulating endothelial-derived and mononuclear-derived progenitor cells in patients with metabolic syndrome. Data Brief. 2016; 8: 717-22.

[14] Berezin AE, Kremzer AA, Martovitskaya YV, Berezina TA, Gromenko EA. Pattern of endothelial progenitor cells and apoptotic endothelial cell-derived microparticles in chronic heart failure patients with preserved and reduced left ventricular ejection fraction. EBioMedicine. 2016; 4: 86-94.

[15] Berezin AE, Kremzer AA. The impact of low-grading inflammation on circulating endothelialderived progenitor cells in patients with metabolic syndrome and diabetes mellitus. Journal of Endocrinology and Diabetes. 2015; 2 (3): 8-16.

[16] Berezin AE, Kremzer AA. Relationship between circulating endothelial progenitor cells and insulin resistance in non-diabetic patients with ischemic chronic heart failure. Diabetes Metab Syndr. 2014; 8(3):138-44.

[17] Berezin AE. Progenitor endothelial cell dysfunction in obese patients: possibilities for cardiovascular risk prediction. Journal of Clinical \& Experimental Cardiology. 2016; 7 (10): 148-150. 
[18] Hur J, Yoon CH, Kim HS, Choi JH, Kang HJ, Hwang KK. et al. Characterization of two types of endothelial progenitor cells and their different contributions to neovasculogenesis. Arterioscler Thromb Vasc Biol. 2004; 24: 288-293.

[19] Lin Y, Weisdorf DJ, Solovey A, Hebbel RP. Origins of circulating endothelial cells and endothelial outgrowth from blood. J Clin Invest. 2000;105:71-77

[20] Peichev M, Naiyer AJ, Pereira D, Zhu Z, Lane WJ, Williams M et al. Expression of VEGFR-2 and $\mathrm{AC} 133$ by circulating human $\mathrm{CD} 34(+)$ cells identifies a population of functional endothelial precursors. Blood. 2000;95:952-958

[21] Arai F, Hirao A, Ohmura M, Sato H, Matsuoka S, Takubo K. et al. Tie2/angiopoietin-1 signaling regulates hematopoietic stem cell quiescence in the bone marrow niche. Cell. 2004; 118(2): 149161.

[22] Yang JX, Pan YY, Ge JH, Chen B, Mao W, Qiu YG, et al. Tanshinone II A Attenuates TNF- $\alpha-$ Induced Expression of VCAM-1 and ICAM-1 in Endothelial Progenitor Cells by Blocking Activation of NF-кB. Cell Physiol Biochem. 2016; 40(1-2):195-206.

[23] Koller L, Hohensinner P, Sulzgruber P, Blum S, Maurer G, Wojta J, Hülsmann M, Niessner A. Prognostic relevance of circulating endothelial progenitor cells in patients with chronic heart failure. Thromb Haemost. 2016; 116(2): 309-16.

[24] Berezin AE, Kremzer AA, Samura TA, Martovitskaya YV, Malinovskiy YV, Oleshko SV, et al. Predictive value of apoptotic microparticles to mononuclear progenitor cells ratio in advanced chronic heart failure patients. J Cardiol. 2015; 65(5): 403-11.

[25] Fadini GP, de Kreutzenberg SV, Coracina A, Baesso I, Agostini C, Tiengo A, et al. Circulating CD34+ cells, metabolic syndrome, and cardiovascular risk. Eur Heart J 2006; 27: 2247-2255.

[26] Berezin AE, Kremzer AA. Analysis of various subsets of circulating mononuclear cells in asymptomatic coronary artery disease. Journal of Clinical Medicine. 2013; 2 (3), 32-44

[27] Berezin AE, Kremzer AA, Martovitskaya YV, Berezina TA, Samura TA. The utility of biomarker risk prediction score in patients with chronic heart failure. Clin Hypertens. 2016; 22: 3.

[28] Yang SW, Hennessy RR, Khosla S, Lennon R, Loeffler D, Sun T, et al. Circulating osteogenic endothelial progenitor cell counts: new biomarker for the severity of coronary artery disease. Int J Cardiol. 2017; 227: 833-839.

[29] Berezin AE, Samura TA, Kremzer AA, Berezina TA, Martovitskaya YV, Gromenko EA. An association of serum vistafin level and number of circulating endothelial progenitor cells in type 2 diabetes mellitus patients. Diabetes Metab Syndr. 2016; 10(4):205-212.

[30] Berezin AE. Biological markers of cardiovascular diseases. Part 3. Diagnostic and prognostic value of biological markers in stratification of patient $\mathrm{s}$ with cardiometabolic risk. Lambert Academic Publishing GmbH, Moscow, 2015.

[31] Berezin AE, Kremzer AA, Samura TA, Martovitskaya YV. Apoptotic microparticles to progenitor mononuclear cells ratio in heart failure: relevance of clinical status and outcomes JCvD. 2014; 2 (2), 50-57.

Citation: Alexander E. Berezin. Could be Used an Immune Phenotypes of Circulating Endothelial Progenitor Cells as a Biomarker of Cardiovascular Disease? ARC Journal of Cardiology. 2017;3(1):9-12.

Copyright: ( 92017 Alexander E. Berezin. This is an open-access article distributed under the terms of the Creative Commons Attribution License, which permits unrestricted use, distribution, and reproduction in any medium, provided the original author and source are credited. 